\title{
Feature-based control of attention: Evidence for two forms of dimension weighting
}

\author{
TAKATSUNE KUMADA \\ National Institute of Bioscience and Human Technology, Tsukuba, Japan
}

\begin{abstract}
In three experiments, I examined whether prior knowledge of a target feature dimension is useful for guiding spatial attention to the target in a variety of tasks: visual search (Experiments 1A and 1B), texture segregation (Experiment 2), and visual enumeration (Experiment 3). Experiment 1A used a simple search task and found that reaction times for blocks in which a target was defined within a single feature dimension were shorter than those for blocks in which a target was defined across dimensions (within-dimension facilitation; WDF). Intertrial facilitation (ITF; Müller, Heller, \& Ziegler, 1995), a dimension-based priming effect from one trial to the immediately following one, was also observed. Both WDF and ITF disappeared when the same stimuli were used under a compound search task (Experiment 1B), in which participants responded to an attribute of the target in a feature dimension different from its defining dimensions. Experiments 2 and 3 showed that WDF and ITF are not necessarily contingent upon each other: In a texture discrimination task, only WDF was found; in an enumeration task for six or seven targets, only ITF was found. These results show that the two forms of dimension weighting, WDF and ITF, are mediated by different mechanisms. WDF was eliminated when focal attention to targets was required, suggesting that feature-based modulation is limited as a source for controlling spatial attention (Kumada, 1999). ITF was correlated with type of response, suggesting dimension-specific response mechanisms (Cohen \& Shoup, 1997).
\end{abstract}

Two-stage models of visual processing (e.g., Broadbent, 1958) consider visual stimuli to be processed in two successive stages. In the first stage, referred to as the preattentive stage, visual stimuli are processed in parallel across the visual field. This first stage is considered to be specialized for analyzing visual features. In the second stage of processing, referred to as the limited-capacity stage, visual information from the first stage is selected and further analyzed. A question concerning the two-stage model of visual processing has been asked repeatedly: What kind of information or knowledge is used to prioritize processing in the limited-capacity stage?

Recently, prior knowledge of which feature dimension defines the target has been shown to facilitate processing in visual search tasks (Found \& Müller, 1996; Müller, Heller, \& Ziegler, 1995; Treisman, 1988). These studies suggested that such top-down information of the targetdefining features modulates activity in the first, featureprocessing stage. Recent models of visual search have also assumed that feature-based top-down influences on feature processing modulate summed activation on the

The author is grateful to Glyn Humphreys for providing a supportive environment during the preparation of this manuscript. The author appreciates the helpful comments made by Eleanor Batchelder, Asher Cohen, Hermann Müller, and an anonymous reviewer. Correspondence concerning this article should be addressed to T. Kumada, Human Informatics Department, National Institute of Bioscience and Human Technology, 1-1 Higashi, Tsukuba, Ibaraki 305-8566, Japan (e-mail: kumada@nibh.go.jp). master map (Treisman \& Sato, 1990; Wolfe 1994), but the precise mechanisms are not well understood. Furthermore, there are conflicting results in which knowledge of the target feature dimension was ineffective for selecting a target (Kumada, 1999; Theeuwes, 1991, 1992).

\section{Cross-Dimensional Interference}

Theeuwes $(1991,1992)$ showed that attending to the target-defining feature dimension is not always effective. In his experiments, participants searched for a target defined by one feature dimension while trying to ignore a singleton distractor defined by another feature dimension irrelevant to the target. Theeuwes found an asymmetric pattern of interference, referred to as cross-dimensional interference. For example, when the target was defined by shape, a color-defined singleton distractor interfered with the search, but a shape-defined singleton distractor did not interfere with the search for a color-defined target. Theeuwes concluded that participants' attention to a feature dimension could not override stimulus-driven interference from the singleton distractor, even when they knew in advance the feature dimension of the target, and that selection of the target was based on the relative saliency of the singleton.

The task used by Theeuwes $(1991,1992)$ was not a simple search task, as is typically used in visual search studies, but a compound search task. This distinction was first made by Duncan (1985), who demonstrated that the compound search task involved processes different from the simple search task. In simple search tasks, participants 
search for a prespecified target in a visual array and respond to its presence or absence. In compound search tasks, the feature that defines the target is different from the feature to be reported. The task is to search for a target that is specified by one feature dimension and to respond to another feature of the target (the to-be reported attribute) when it is found. Duncan argued that the targetdefining attribute is analyzed in the preattentive stage and that, in simple search tasks, participants respond on the basis of the output of this stage. On the other hand, in compound search tasks, the to-be-reported attribute is analyzed in the limited-capacity stage by focusing attention on the target after locating it, so a response requires analysis in this second stage. Results obtained in compound search tasks have been difficult to apply to models of simple visual search, since different processes are involved in each task.

To create a theoretical linkage between compound search studies and typical visual search studies, Kumada (1999) compared cross-dimensional interference in simple search tasks and compound search tasks, using similar stimulus displays and target conditions. In compound search tasks with targets defined by color or orientation and singleton distractors, asymmetrical cross-dimensional interference was found. However, in simple search tasks, in which the participant's task was to respond only to the presence or absence of a target, there was no significant effect of a singleton distractor, in either color or orientation target conditions. The cross-dimensional interference effect from a salient irrelevant singleton distractor was eliminated under a simple search task, in which participants were able to use prior knowledge of the target feature dimension to override stimulus-driven cross-dimensional interference. The different pattern of results between the simple and the compound search tasks shows that the effectiveness of prior knowledge of the target-defining feature dimension depends on the task.

The task-dependent cross-dimensional interference shown by Kumada (1999) can also be explained by assuming that the different tasks draw upon different processing stages. Knowledge of the target-defining feature dimension was effective when responses were elicited in the preattentive stage, without involving the limitedcapacity stage, but the knowledge was not useful when both the preattentive and the limited-capacity stages were engaged in processing. These results lead to the hypothesis that knowledge of a target-defining feature dimension is useful for the detection of a target that can be processed mainly in the preattentive stage but the same knowledge does not affect processing in the limited-capacity system. The purpose of the present study is to test this hypothesis by using another effect, which I refer to as withindimension facilitation (WDF).

\section{Within-Dimension Facilitation}

Several studies have reported that prior knowledge of the target-defining feature dimension facilitated detec- tion of the target (Found \& Müller, 1996; Müller et al., 1995; Treisman, 1988). Treisman presented a feature search task in which targets were defined by color, orientation, or size. Each experimental block consisted of stimuli in one of three conditions. In the target-known condition, both the feature dimension and the particular feature value that defined the targets were fixed within a block of trials (e.g., a blue bar). Reaction times (RTs) in this condition were almost the same as those in the withindimension condition, in which targets were defined by varying feature values within a single dimension (e.g., a blue, red, or white bar among green bars). In the crossdimension condition, however, in which targets were defined by any one of three feature dimensions (color, orientation, or size), there was a significant increase in RTs (90 msec), relative to the within-dimension condition.

This facilitation of the within-dimension condition relative to the cross-dimension condition is the effect that I refer to as WDF. Recently, Müller and colleagues (Found \& Müller, 1996; Müller et al., 1995) also found this effect, attributing it to knowledge of the target-defining feature dimensions. They referred to the mechanism that produces feature-based WDF in feature search tasks as the dimension-weighting mechanism. Their explanatory framework agrees with the Guided Search model (Wolfe, 1994), but Found and Müller claimed that greater weight is assigned to known feature dimensions, relative to other dimensions. The amplified dimensional signal from this dimension-weighting mechanism produces strong activation of target locations on the master map, generating rapid responses. Found and Müller also showed that the weighting on a feature dimension persists across consecutive trials; RTs are faster to a target that is defined by the same dimension as that in the immediately preceding trial, rather than by a different dimension. This effect is called intertrial facilitation (ITF).

\section{Rationale for This Study}

Although these previous studies have documented a WDF effect, all these studies used simple search tasks. The hypothesis presented in this study is that knowledge of a target feature dimension can be useful for detecting the target in the preattentive stage but cannot be the basis for guiding spatial attention to the target in the limitedcapacity stage. The results of dimension-weighting studies have supported part of this hypothesis: Feature knowledge was found to be advantageous in simple search tasks, which are thought to involve only the preattentive stage. My hypothesis further predicts that WDF will be eliminated under compound search tasks, which require processing in the limited-capacity stage also.

Most recently, Cohen and Magen (1999) reported that within-dimension facilitation was eliminated under one type of compound search task. ${ }^{1}$ In their Experiment 1, there were three types of tasks: orientation, color, and cross-dimension. In the orientation task, a target was defined by a difference along the orientation dimension, and 
participants responded to the orientation of the target (left-tilted or right-tilted) by pressing one of two keys. In the color task, participants discriminated between targets of two different colors. In the cross-dimension task, participants were required to make one response when the target was green and another when it was right-tilted. Cohen and Magen found no advantage in RTs in the withindimension condition, relative to the cross-dimension condition. These results seem to be consistent with my prediction that WDF will not be found in a compound search task. However, Cohen and Magen explained the results with a different theory, proposed by Cohen and Shoup (1997), that assumed individual selection mechanisms for each feature module. Cohen and Magen argued that it was manipulation of the stimulus-to-response mapping that had the strong effect of eliminating WDF, so facilitation should be attributed to response selection processes.

One procedural aspect of Cohen and Magen's (1999) study prevents me from applying their results directly to my hypothesis. In their study, to-be-reported attributes were not the same in the two within-dimension conditions and the cross-dimension condition. In the within-dimension conditions, the feature value of the target had to be determined in order to make responses, but in the crossdimension condition, participants could respond by simply noting the target-defining feature dimension-that is, by checking for the presence of a feature singleton on feature maps-without accessing the target feature value. It thus seems possible that limited-capacity stage processes were involved in the within-dimension conditions, but not in the cross-dimension condition. Therefore, my hypothesis cannot be evaluated in terms of these results. In Experiment 1, I looked at WDF, using a compound search task in which the to-be-reported attributes were the same in both the within- and the cross-dimension conditions.

\section{EXPERIMENT 1A}

\section{Simple Search Task}

Experiment 1 was designed to test whether target knowledge is equally effective in a simple search task (Experiment 1A) and a compound search task (Experiment 1B). Experiment 1A replicated experiments reported in Treisman (1988) and Müller et al. (1995, Experiment 1), with participants being asked to detect oddball targets defined by color, orientation, or size. Since both studies had reported WDF in a simple search task, a similar pattern of facilitation was expected here. In Experiment 1B, the same displays as those in Experiment $1 \mathrm{~A}$ were presented under a compound search task, with participants responding to a secondary attribute of the target in both withinand cross-dimension conditions. My hypothesis predicted the elimination of WDF in this compound search task.

\section{Method}

Participants. Eight undergraduate students (5 males and 3 females; $20-23$ years of age) participated in the experiment as paid
(A) Orientation (left-oriented) target

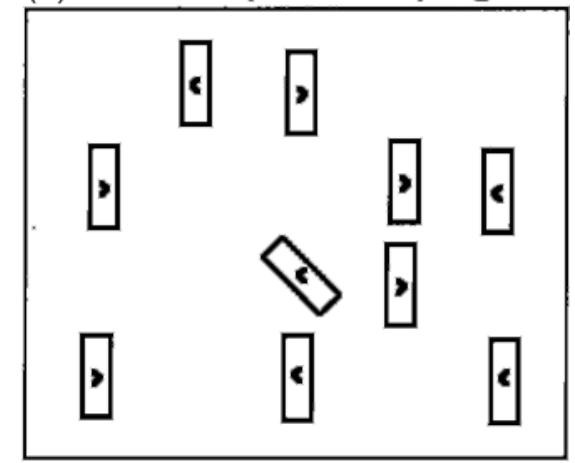

(B) Color target

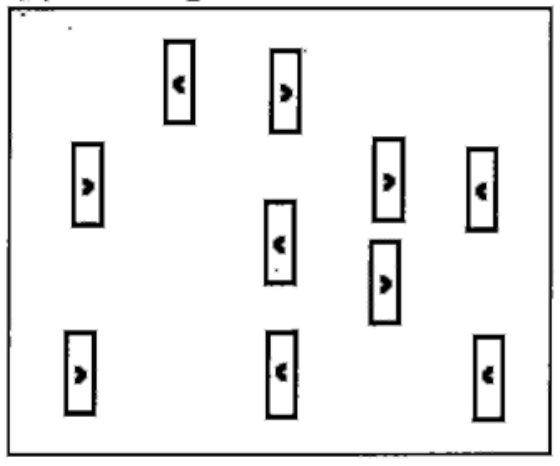

(C) Size target

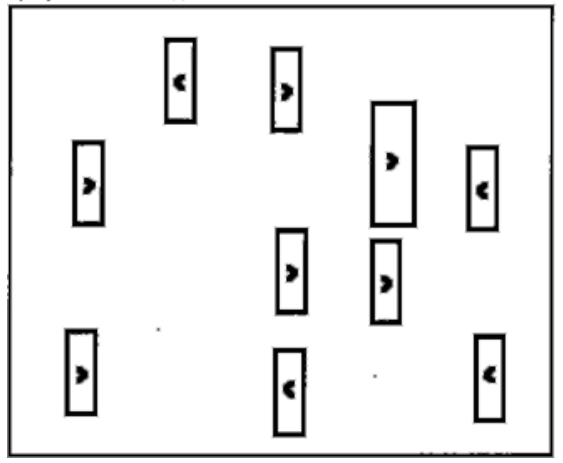

Figure 1. Examples of stimulus displays in Experiment 1: (A) orientation target, (B) color target, and (C) size target conditions. All the stimuli were presented gray on a black background, except for color targets, which were drawn in green.

volunteers. All had normal or corrected-to-normal visual acuity and normal color vision.

Apparatus and Stimuli. Visual stimuli were generated and controlled by a computer (Apple Power Macintosh 8500) and presented on a 16-in. color CRT display (Sony high-resolution color display).

Figure 1 shows a sample display. The stimulus elements were either small $(12 \times 4 \mathrm{~mm})$ or large $(18 \times 6 \mathrm{~mm})$ rectangles. Each stimulus element contained a left-pointing or a right-pointing L-shape, each leg $1.5 \mathrm{~mm}$ long. Ten elements were randomly scattered in cells of an imaginary $4 \times 5$ matrix $\left(80 \times 100 \mathrm{~mm} ; 8^{\circ} \times 10^{\circ}\right.$ in visual angle). In target-absent trials, 10 nontargets of small gray vertical rectangles were presented. In target-present trials, 1 of the 
10 nontargets was replaced by a target. There were three types of target def inition: control, within-dimension, and cross-dimension. In the control condition, a target was a small gray left-oriented rectangle (rotated $45^{\circ}$ counterclockwise from the vertical). In the withindimension condition, a target was a small gray rectangle that was horizontal, left-oriented, or right-oriented. In the cross-dimension condition, a target was a small gray left-oriented rectangle, a small green vertical one, or a large gray vertical one.

The participants received two blocks of control, four blocks of within-dimension and four blocks of cross-dimension conditions. Each block consisted of 96 trials presented in random order. In half of the trials in each block, a target was presented, and in the other half of the trials, a target was absent. In the within- and cross-dimension conditions, 16 trials each of the three types of targets were presented as target-present trials. The order of presentation of the blocks was counterbalanced across participants. Before the first block of each condition, the participants received a practice block of 30 trials.

Procedure. The participants were seated $57 \mathrm{~cm}$ from the CRT display, with their heads supported by a chinrest. The sequence of each trial was as follows: First, a small white dot was presented as a fixation point in the center of the display for $1,000 \mathrm{msec}$. After $200 \mathrm{msec}$ of a blank display, a stimulus display was presented and remained until the participant responded, followed by an intertrial interval of $500 \mathrm{msec}$. The time from onset of the presentation of the stimulus display to initiation of a response was measured as the RT in each trial. The participant's task was to search for an oddball target and to report the presence or absence of the target as quickly and accurately as possible by pressing one of two keys, one key with their right index finger if a target was present or another key with their left index finger if no target could be found.

\section{Results}

RT and error analyses. In this and all the subsequent experiments reported in this article, RT outliers were removed from the data set prior to analysis. Outliers were defined by the modified recursive outlier elimination procedure (Van Selst \& Jolicœur, 1994). In Experiment 1A, this resulted in the removal of $3.5 \%$ of all the the observations. For each target-definition condition, only trials with left-oriented targets and with target absent were analyzed.

Mean correct RTs are shown in Table 1. A two-way analysis of variance (ANOVA) was performed, and the main effects of both target definition condition (control/ within/cross) and response (target present/absent) were significant $[F(2,14)=53.72, p<.0001$, and $F(1,7)=$ $7.86, p<.05$, respectively]. The two-way interaction was also significant $[F(2,14)=19.61, p<.0001]$. Planned comparisons between target definition conditions for target-present trials showed that, although the difference between control and within-dimension conditions was not significant $[F(1,14)=3.69, p=.075]$, the other two

Table 1

Mean Correct Reaction Times (RTs, in Milliseconds) and Error Rates (PEs, Percentages) for Experiment 1A

\begin{tabular}{lccccc}
\hline & \multicolumn{4}{c}{ Target Response } \\
\cline { 2 - 3 } \cline { 5 - 6 } Target Definition & RT & PE & & RT & PE \\
\cline { 2 - 3 } \cline { 5 - 6 } Control & 406.8 & 1.95 & & 427.1 & 2.99 \\
Within-dimension & 417.3 & 2.15 & & 455.0 & 2.34 \\
Cross-dimension & 447.1 & 2.54 & & 515.1 & 2.47 \\
\hline
\end{tabular}

Table 2

Mean Correct Reaction Times (RTs) in Trial $\boldsymbol{n}$ and Intertrial Facilitation (ITF; Both in Milliseconds) for OrientationDefined Targets as a Function of the Target-Defining Dimension in the Previous Trial $(n-1)$ in Experiment $1 \mathrm{~A}$

\begin{tabular}{lcc}
\hline $\begin{array}{l}\text { Target-Defining Dimension } \\
\text { of Trial } n-1\end{array}$ & RT & ITF \\
\hline Orientation & 421.6 & \\
Color & 445.8 & $24.2 \dagger$ \\
Size & 453.9 & $32.3^{*}$ \\
Average of color and size & 449.8 & $28.2^{*}$ \\
\hline
\end{tabular}

${ }^{*} p<.05 . \quad \dagger .05<p<.10$.

comparisons reached significance [control and crossdimension, $F(1,14)=54.53, p<.0001$; within- and cross-dimension, $F(1,14)=29.84, p<.0001]$. RTs in the cross-dimension condition were longer than those in the control (by $40.3 \mathrm{msec}$ ) and within-dimension (by $29.8 \mathrm{msec}$ ) conditions. This pattern of results is consistent with previous studies that showed WDF (Müller et al., 1995; Treisman, 1988).

Table 1 also shows the error rates for each condition. In this and all the subsequent experiments reported in this article, the error rates were subjected to an arc-sine transformation before being entered into an ANOVA. A two-way (target definition $\times$ response) ANOVA showed that both of the main effects and their interaction were not significant (all $F \mathrm{~s}<1)$. Error rates remained constant across all conditions, suggesting that the participants did not tend to make a speed-accuracy tradeoff.

Intertrial facilitation. For the cross-dimension condition, correct RTs to a target on a given trial $n$ were categorized by the defining dimension of the target in the immediately previous trial $(n-1)$. Trials following an incorrect response were not included in this analysis. Table 2 shows the means of correct RTs to orientation-defined targets categorized by the target dimension in trial $n-1$. A one-way ANOVA was performed, giving a significant main effect of dimension in trial $n-1[F(2,14)=4.26$, $p<.05]$. Planned comparisons showed significant or marginally significant differences between RTs in orientation trials preceded by orientation trials and RTs in those preceded by other target trials: [orientation and color, $F(1,14)=4.40, p=.055$; orientation and size, $F(1,14)=$ $7.87, p<.05$; orientation and average of color and size, $F(1,14)=8.01, p<.05]$. RTs were, on average, $28.2 \mathrm{msec}$ faster when the displays contained a target defined by the same dimension as that in the previous trial than when defined by a different dimension. This pattern of ITF was identical to that in the previous study (Found \& Müller, 1996, Experiment 1).

\section{Discussion}

In Experiment 1A, there was clear WDF, consistent with previous studies reporting knowledge-based facilitation of a feature search (Müller et al., 1995; Treisman, 1988). Intertrial analysis showed a pattern of ITF that also was consistent with a previous study (Found \& Müller, 1996). Experiment 1A thus established that the 
displays used were sufficient for showing WDF and ITF when presented under a simple search task.

\section{EXPERIMENT 1B Compound Search Task}

The same stimulus displays as those in Experiment $1 \mathrm{~A}$ were presented under a compound search task, which required spatial attention to be focused on the location of the target. My hypothesis predicted that the facilitatory effect of prior knowledge of target-defining feature dimensions would be eliminated, because such knowledge would not be useful for guiding spatial attention.

\section{Method}

Participants. Eight undergraduate students (4 males and 4 females; $19-22$ years of age) participated as paid volunteers. All had normal or corrected-to-normal visual acuity and normal color vision, and none had participated in Experiment 1A.

Apparatus and Stimuli. The apparatus and stimuli were the same as those used in Experiment 1A, except that a target was present in all the trials and, in addition to 10-element trials, 1-element trials that contained no nontargets were also presented. Two numberof-elements conditions (1 and 10) and 3 target def inition conditions (control, within-dimension, and cross-dimension) were presented in separate blocks. The participants received 10 blocks of trials: 1 block each of the control condition with 1 element and 10 elements, 2 blocks each of the within-dimension condition with 1 element and 10 elements, and 2 blocks each of the cross-dimension condition with 1 element and 10 elements. Each block consisted of 96 trials. In the within- and cross-dimension conditions, 32 trials of each of the three types of targets were presented. In half of the trials, the target had a left-pointing L-shape, and in the other half, a right-pointing L-shape (see Figure 1). The order of presentation of the 10 blocks was counterbalanced across participants. Before the 1 st block of each pairing of target definition condition and number-of-elements condition, the participants received a practice block of 30 trials (6 practice blocks in all).

Procedure. The procedure was the same as that in Experiment 1A, except that the task was to search for the target and then to respond to the orientation of its L-shape (the to-be-reported attribute) as quickly and accurately as possible by pressing one of two keys, one key with the right index finger if the L-shape was pointing to the right $(>)$ and another key with the left index finger if it was pointing to the left $(<)$.

\section{Results}

RT and Error Analyses. RT outliers were $1.7 \%$ of all observations.

Mean correct RTs for the left-oriented targets are shown in Table 3. A two-way ANOVA was performed, and both main effects were significant ${ }^{2}$ [target definition condition, $F(2,14)=6.50, p<.05$; number-of-elements con-

Table 3

Mean Correct Reaction Times (RTs, in Milliseconds) and Error Rates (PEs, Percentages) for Experiment 1B

\begin{tabular}{lccccc}
\hline & \multicolumn{3}{c}{ Number of Elements } \\
\cline { 2 - 3 } \cline { 5 - 6 } Target Definition & RT & PE & & RT & PE \\
\cline { 2 - 3 } \cline { 5 - 6 } Control & 635.1 & 4.69 & & 724.3 & 4.69 \\
Within-dimension & 647.8 & 4.88 & & 746.5 & 4.30 \\
Cross-dimension & 643.1 & 4.88 & & 752.5 & 6.84 \\
\hline
\end{tabular}

Table 4

Mean Correct Reaction Times (RTs) in Trial $n$ and Intertrial Facilitation (ITF; Both in Milliseconds) for OrientationDefined Targets as a Function of the Target-Defining Dimension in the Previous Trial $(n-1)$ in Experiment 1B

\begin{tabular}{lccccr}
\hline \multirow{2}{*}{$\begin{array}{l}\text { Target-Defining Dimension } \\
\text { of Trial } n-1\end{array}$} & \multicolumn{2}{c}{1 Element } & & \multicolumn{2}{c}{10 Elements } \\
\cline { 2 - 3 } & RT & ITF & & RT & ITF \\
\hline Orientation & 633.3 & & & 748.5 & \\
Color & 646.0 & 12.7 & & 743.4 & -5.2 \\
Size & 649.5 & 16.2 & & 754.8 & 6.3 \\
Average of color and size & 647.7 & 14.4 & & 749.1 & 0.6 \\
\hline
\end{tabular}

dition, $F(1,7)=41.16, p<.001]$. The two-way interaction was not significant $(F<1)$. Planned comparisons between target-definition conditions showed significant differences [control and within-dimension conditions, $F(1,14)=9.40, p<.01 ;$ control and cross-dimension conditions, $F(1,14)=10.10, p<.01]$. However, there was no significant difference between the within-dimension and the cross-dimension conditions $(F<1)$, indicating that there was no WDF effect.

Table 3 also shows the error rates for each condition. A two-way (target definition $\times$ number of elements) ANOVA showed that both of the main effects and their interaction were not significant (all $p$ s $>.14$ ). Error rates were constant across all conditions, suggesting that the participants did not tend to make a speed-accuracy tradeoff.

Intertrial facilitation. For the cross-dimension condition, ITF was analyzed by the same method as that in Experiment 1A. Table 4 shows the means of correct RTs in trial $n$ categorized by the target-defining dimension in trial $n-1$. A two-way ANOVA was performed with the main terms of dimension in trial $n-1$ and number of elements. The main effect of number of elements was significant $[F(1,7)=15.69, p<.01]$. Neither the main effect of dimension nor the dimension $\times$ number of elements interaction was significant (all $F_{\mathrm{s}}<1$ ). This result clearly shows that there was no ITF effect.

\section{Discussion}

When the simple search task was replaced by a compound search task, both WDF and ITF disappeared. These results are consistent with my prediction that the facilitatory effect of prior knowledge of the target-defining feature dimension would not be observed under a compound search task.

\section{DISCUSSION OF EXPERIMENTS 1A AND 1B}

The results of these experiments are clear-cut. When a simple search task was used (Experiment 1A), both WDF and ITF were seen, but when a compound search task was used (Experiment 1B), both types of facilitation were eliminated. This pattern of results is consistent with the prediction that prior knowledge is useful under a simple search task, but not under a compound search task. This prediction was derived from the hypothesis that feature dimensions weighted by knowledge are not available to guide spatial attention to the target location. In- 
stead, I suggest that spatial attention is guided simply by a saliency signal derived from the unweighted sum of activation in feature maps. I refer to this as the attentional limitation account.

The results of Experiments $1 \mathrm{~A}$ and $1 \mathrm{~B}$ also seem to be consistent with the response account of Cohen and Magen (1999), according to which search processes in simple search tasks are the same as those in compound search tasks and the absence of WDF in the compound search task is instead due to modification of the to-be-reported features. In the present experiments, it could be said that the detection responses in the simple search task (Experiment $1 \mathrm{~A}$ ) were elicited directly by dimension-specific response selection processes but that the discrimination responses in the compound search task (Experiment 1B) required additional processes for selecting among possible responses and these processes eliminated WDF.

\section{EXPERIMENT 2 Texture Segregation Tasks}

A critical test to differentiate the attentional limitation account from the response account would be a task in which discrimination responses are required, just as in Experiment 1B, but in which focusing of attention is not necessary to locate the target. Texture segregation is a task that has been considered not to involve focal attention (e.g., Nothdurft, 1992). In a display of many similar objects, a region is defined by a difference in a single feature and is perceived as clearly segregated from a background region. Assuming that these regions could be segregated preattentively, the global properties of the display could be detected without focusing attention on a particular spatial region.

In Experiment 2, two texture segregation tasks were performed, using identical display sets. A texture boundary was defined by one dimension in the within-dimension condition and by one of three dimensions in the crossdimension condition (see Figure 2). In the detection task, the participants responded to the presence or absence of a texture boundary. In the discrimination task, the par- ticipants responded to the orientation of the texture boundary (vertical or horizontal).

The response account predicts a pattern of results similar to that in Experiment 1: WDF in the detection tasks, but no WDF in the discrimination tasks, owing to the need for additional response selection processes. The attentional limitation account predicts that there will be WDF in both detection and discrimination tasks, because in both tasks participants can respond without focusing their attention on a particular spatial region of the display. In other words, this account claims that knowledge of feature dimensions will be available in both tasks, since limited-capacity processes are not required either for detecting or for discriminating the texture boundary.

\section{Method}

Participants. Eight undergraduate students (6 males and 2 females; $22-24$ years of age) participated as paid volunteers. All had normal or corrected-to-normal visual acuity and normal color vision, and none had participated in Experiment 1.

Stimuli. Stimulus elements were small $(12 \times 4 \mathrm{~mm})$ or large $(18$ $\times 6 \mathrm{~mm})$ rectangles. The elements filled in an imaginary $6 \times 6$ matrix $\left(120 \times 120 \mathrm{~mm} ; 12^{\circ} \times 12^{\circ}\right.$ in visual angle). Arrays of two types of elements created a texture boundary either horizontally or vertically (Figure 2). In the horizontal boundary condition, one type of element occupied the three rows above the fixation point, and elements of a different type occupied the three lower rows. In the vertical boundary condition, one type of element occupied the three columns on the left of the fixation point, with others on the right. The texture boundary was defined by a color, orientation, or size dimension. In all the conditions, one of the two types of elements was always small white vertical rectangles. In the color condition, the other elements were small green vertical rectangles. In the orientation condition, the other elements were small white rectangles with one of three orientations: left-tilted, horizontal, or right-tilted. In the size condition, the other elements were large white vertical rectangles. In addition, a boundary-absent display was used, in which all the elements were small white vertical rectangles.

As in Experiment 1A, three types of target definition (control, within-dimension, and cross-dimension ) were presented in a boundary detection task and a discrimination task. In the control condition, texture boundaries were always created by left-tilted elements. In the within-dimension condition, texture boundaries were defined by left-tilted, vertical, or right-tilted orientation. In the cross-dimension condition, texture boundaries were defined by color, orientation
(A)

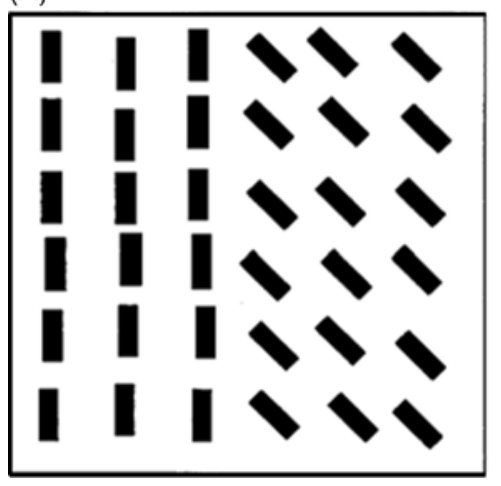

(B)

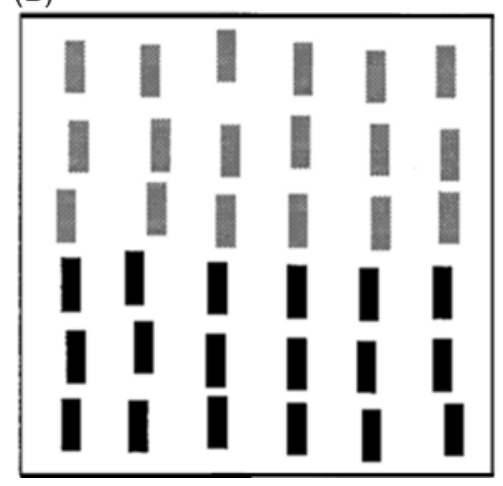

(C)

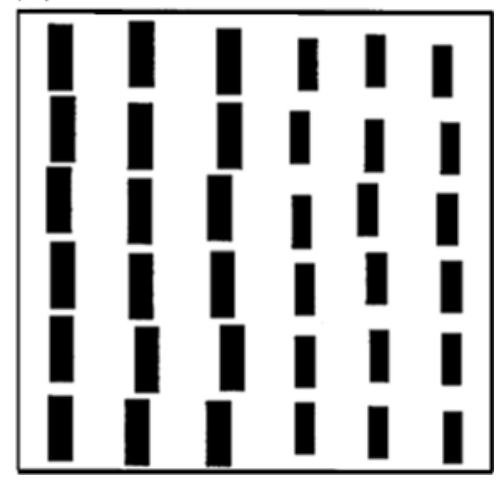

Figure 2. Example of stimulus displays in Experiment 2: (A) orientation target, (B) color target, and (C) size target conditions. 
(left-tilted), or size. In both within- and cross-dimension conditions, the three different types of target were presented with equal probability. In the detection task, a texture boundary was presented (either horizontal or vertical) in half of the trials, and boundary-absent displays in the other half. In the discrimination task, half of the trials had a horizontal boundary, and the other half had a vertical boundary.

Each participant received 2 blocks of control, 4 blocks of withindimension, and 4 blocks of cross-dimension conditions for each of the two tasks. Each block was composed of 96 trials in the detection task and 48 trials in the discrimination task. Half of the participants started with 10 blocks of the detection task, and the other half started with 10 blocks of the discrimination task. Before the 1st block of each target definition condition of each task, the participants received a practice block of 30 trials ( 6 practice blocks in all).

Procedure. The procedure was the same as that in Experiments $1 \mathrm{~A}$ and $1 \mathrm{~B}$, except for the tasks. In the detection task, the participants responded to the presence or absence of a texture boundary by pressing one of two keys. In the discrimination task, the participants responded to the orientation of the texture boundary (vertical or horizontal) by pressing one of two keys.

\section{Results}

Detection task. RT outliers were $4.3 \%$ of all observations. Trials with a boundary defined by left-tilted elements (boundary-present) and trials with no texture boundary (boundary-absent) were analyzed.

Mean correct RTs are shown in Table 5. A two-way ANOVA was performed with main terms of target definition and response, and both main effects were significant $[F(2,14)=67.08, p<.0001$, and $F(1,7)=9.92, p<$ .05 , respectively]. The two-way interaction was not significant $(p=.220)$. Planned comparisons were conducted of combinations of target definitions, and all three comparisons showed significant differences [control and within-dimension conditions, $F(1,14)=4.78, p<.05$; control and cross-dimension conditions, $F(1,14)=$ $119.78, p<.0001$; within- and cross-dimension conditions, $F(1,14)=76.68, p<.0001]$. WDF $(29.6 \mathrm{msec})$ was quite similar to that in Experiment $1 \mathrm{~A}$.

Table 5 also shows the error rates for each condition. A two-way (target definition $\times$ response) ANOVA showed that both of the main effects and their interaction were not significant $\left(\right.$ all $\left.F_{\mathrm{S}}<1\right)$. Error rates were constant across all conditions, suggesting that the participants did not tend to make a speed-accuracy tradeoff.

For the cross-dimension condition, ITF was analyzed by the same method as that in Experiment 1A. Table 6 shows the means of correct RTs in trial $n$ categorized by the target-defining dimension in trial $n-1$. A one-way

Table 5

Mean Correct Reaction Times (RTs, in Milliseconds) and Error Rates (PEs, Percentages) for the Detection Task in Experiment 2

\begin{tabular}{|c|c|c|c|c|}
\hline \multirow[b]{3}{*}{ Target Definition } & \multicolumn{4}{|c|}{ Texture Boundary Response } \\
\hline & \multicolumn{2}{|c|}{ Present } & \multicolumn{2}{|c|}{ Absent } \\
\hline & RT & $\overline{\mathrm{PE}}$ & RT & $\mathrm{PE}$ \\
\hline Control & 385.8 & 1.63 & 403.5 & 1.50 \\
\hline Within-dimension & 393.1 & 1.75 & 413.7 & 4.50 \\
\hline Cross-dimension & 422.7 & 1.50 & 453.9 & 4.88 \\
\hline
\end{tabular}

Table 6

Mean Correct Reaction Times (RTs) in Trial $\boldsymbol{n}$ and intertrial Facilitation (ITF; Both in Milliseconds) for OrientationDefined Targets as a Function of the Target-Defining Dimension in the Previous Trial $(n-1)$ in Experiment 2

\begin{tabular}{lccccc}
\hline \multirow{2}{*}{$\begin{array}{l}\text { Target-Defining Dimension } \\
\text { of Trial } n-1\end{array}$} & \multicolumn{2}{c}{ Detection Task } & & \multicolumn{2}{c}{ Discrimination Task } \\
\cline { 2 - 3 } & RT & ITF & & RT & ITF \\
\hline Orientation & 410.0 & & & 484.2 & \\
Color & 435.8 & $25.8^{*}$ & & 491.9 & 7.8 \\
Size & 435.9 & $25.9^{*}$ & & 491.2 & 7.0 \\
Average of color and size & 435.9 & $25.9^{*}$ & 491.5 & 7.4 \\
\hline
\end{tabular}

$* p<.05$.

ANOVA showed a significant main effect of the dimension in trial $n-1[F(2,14)=4.40, p<.05]$. Planned comparisons between orientation-boundary trials and other boundary trials showed significant differences [orientation and color, $F(1,14)=6.60, p<.05$; orientation and size, $F(1,14)=6.59, p<.05$; orientation and average of color and size, $F(1,14)=8.79, p<.05]$. There was a significant ITF effect of $25.9 \mathrm{msec}$.

Discrimination task. RT outliers were $4.7 \%$ of all the observations. Trials with a boundary defined by lefttilted elements were analyzed.

Mean correct RTs are shown in Table 7. A one-way ANOVA showed that the main effect of target definition was significant $[F(2,14)=9.61, p<.005]$. A planned comparison showed that the cross-dimension condition was significantly slower than other conditions [control and cross-dimension conditions, $58.5 \mathrm{msec}, F(1,14)=$ $16.25, p<.005$; within- and cross-dimension conditions, $50.9 \mathrm{msec}, F(1,14)=12.30, p<.05]$. There was no significant difference between the control and the withindimension conditions $(p>.6)$. Thus, a considerable WDF effect was observed.

Table 7 also shows the error rates for each condition. A one-way ANOVA, with a main term of target definition, showed that the main effect was not reliable $(F<1)$. Error rates were constant across all the conditions, suggesting that the participants did not tend to make a speed-accuracy tradeoff.

For the cross-dimension condition, ITF was analyzed by the same method as that in Experiment 1A. Table 6 shows the means of correct RTs in trial $n$ categorized by the target-defining dimension in trial $n-1$. A one-way ANOVA performed with the main term of the dimension in trial $n-1$ was not significant $(F<1)$, showing that there was no ITF effect in this task.

\section{Discussion}

In both the detection and the discrimination tasks, there was a significant effect of WDF, which contradicts the prediction of the response account. Although the stimulusto-response mapping was varied between the two tasks, similar to the variation between the simple search task and the compound search task in Experiments 1A and 1B, in this case the change in mapping did not eliminate the WDF effect. 
Table 7

Mean Correct Reaction Times (RTs, in Milliseconds) and Error Rates (PEs, Percentages) for the Discrimination Task in Experiment 2

\begin{tabular}{lcc}
\hline Target Definition & RT & PE \\
\hline Control & 441.2 & 3.38 \\
Within-dimension & 448.8 & 3.13 \\
Cross-dimension & 499.7 & 4.00 \\
\hline
\end{tabular}

However, this result is consistent with the attentional limitation account of WDF. The main difference between the compound search task in Experiment 1B and the texture discrimination task here was that focusing of attention on the target was required in the former, but not in the latter. According to the attentional limitation account, when no focusing of spatial attention on a particular region of the display is required, the weighted signal from feature maps is available for determining responses, even when the responses are confined to discrimination.

In this experiment, the ITF effect showed a different pattern from that of WDF: ITF was observed only in the detection task, ${ }^{3}$ suggesting that ITF and WDF are not necessarily mediated by the same processes. Taken together with the results of Experiments $1 \mathrm{~A}$ and $1 \mathrm{~B}$, ITF is seen to be sensitive to the stimulus-to-response mapping. That is, only when the participants made detection responses did the ITF effect emerge.

In Experiment 3, I seek converging evidence for the attentional limitation account of WDF, using an enumeration task.

\section{EXPERIMENT 3 Enumeration Tasks}

Recent studies have suggested that when a relatively small number of elements are to be enumerated, processes known as subitizing are involved, similar to those that produce visual pop-out (Trick \& Enns, 1997). On the other hand, when a larger number of elements, typically more than four, are to be enumerated, item-by-item counting is used, and serial guidance of spatial attention is considered to be engaged (Trick \& Pylyshyn, 1993, 1994). Experiment 3 used tasks expected to invoke either subitizing or counting processes. In both kind of tasks, the participants discriminated two or three targets in the subitizing task and six or seven in the counting task.

The purpose of this experiment was twofold. First, this experiment examined whether ITF is sensitive to the nature of responses. In Experiments 1B and 2, in which discrimination responses were required, ITF disappeared. In this experiment, if ITF is sensitive to the nature of responses, the ITF effect would be eliminated in both subitizing and counting tasks, because both require discrimination responses of numerosity, rather than simple detection responses. Second, the attentional limitation account of WDF was further tested. The attentional limitation account predicted that WDF would occur only in the subitizing task, since this task, but not the counting task, can be performed without focusing spatial attention on a specific region.

\section{Method}

Participants. Sixteen undergraduate or graduate students (9 males and 7 females; 20-27 years of age) participated as paid volunteers. All had normal or corrected-to-normal visual acuity and normal color vision, and none had participated in Experiments 1 or 2.

Stimuli. The stimulus elements were the same as those in Experiment 2 . Each display consisted of 20 elements, randomly assigned in cells of an imaginary $5 \times 6$ matrix $\left(100 \times 120 \mathrm{~mm} ; 8^{\circ} \times\right.$ $10^{\circ}$ in visual angle). Small gray vertical rectangles were presented as nontargets. There were two tasks. In the subitizing task, two or three nontargets were replaced by targets. In the counting task, six or seven nontargets were replaced by targets. In a given display, all the targets were identical. The definition of targets was similar to those in Experiment 1A. In the within-dimension condition, the targets were small gray rectangles either left-oriented, horizontal, or rightoriented. In the cross-dimension condition, the targets were small gray left-oriented, small green vertical, or large gray vertical rectangles.

The participants received four blocks of within-dimension and four blocks of cross-dimension conditions for each task. Each block was composed of 96 trials, 32 trials each of the three types of targets. Half of the participants started with eight blocks of the subitizing task, and the other half started with the counting task. Before the first block for each target definition condition of each task, the participants received a practice block of 30 trials (four practice blocks in all).

Procedure. The procedure was the same as that in Experiment 1A, except for the tasks. The participants responded to the number of targets by pressing one of two keys, representing a count of two or three in the subitizing task, and a count of six or seven in the counting task. In both tasks, the response indicating the smaller number was assigned to the left hand.

\section{Results}

RT outliers were $2.3 \%$ of all observations. Trials with left-oriented targets were analyzed.

Subitizing task. Mean correct RTs are shown in Table 8. A two-way ANOVA was performed with main terms of target definition and response (two or three), and both main effects were significant $[F(1,15)=31.522$, $p<.0001$, and $F(1,15)=25.38, p<.0001$, respectively]. The two-way interaction was not significant $(p=.755)$.

Table 8 also shows the error rates for each condition. A two-way (target definition $\times$ response) ANOVA showed that the main effect of response was significant $[F(1,15)=$ $10.16, p<.01]$. The main effect of target definition and the target definition $\times$ response interaction were not significant $(p s>.2$ ), even though the participants made more errors in three-response trials than in two-response trials. This suggests that the difference in RTs between

Table 8

Mean Correct RTs (in Milliseconds) and Error Rates (PE, Percentages) for the Subitizing Task in Experiment 3

\begin{tabular}{lccccc}
\hline & \multicolumn{4}{c}{ Response } \\
\cline { 2 - 3 } & \multicolumn{2}{c}{ Two Targets } & & \multicolumn{2}{c}{ Three Targets } \\
\cline { 2 - 3 } \cline { 5 - 6 } Target Definition & RT & PE & & RT & PE \\
\hline Within-dimension & 547.8 & 0.94 & & 519.4 & 2.25 \\
Cross-dimension & 606.0 & 0.69 & & 574.4 & 2.50 \\
\hline
\end{tabular}


Table 9

Mean Correct Reaction Times (RTs) in Trial $\boldsymbol{n}$ and Intertrial Facilitation (ITF ; Both in Milliseconds) for OrientationDefined Targets as a Function of the Target-Defining Dimension in the Previous Trial $(n-1)$ in the Subitizing Task of Experiment 3

\begin{tabular}{lccccc}
\hline \multirow{2}{*}{$\begin{array}{l}\text { Target-Defining Dimension } \\
\text { of Trial } n-1\end{array}$} & \multicolumn{2}{c}{ Two Targets } & & \multicolumn{2}{c}{ Three Targets } \\
\cline { 2 - 3 } & RT & ITF & & RT & ITF \\
\hline Orientation & 585.2 & & & 554.8 & \\
Color & 609.1 & $23.9^{*}$ & & 573.1 & $18.3 \dagger$ \\
Size & 619.9 & $34.7^{*}$ & & 592.4 & $37.6^{*}$ \\
Average of color and size & 614.5 & $29.3^{*}$ & & 582.7 & $27.9^{*}$ \\
\hline
\end{tabular}

$* p<.05 . \dagger .05<p<.10$.

target definition conditions was not due to a speedaccuracy tradeoff.

For the cross-dimension condition, ITF was analyzed by the same method as that in Experiment $1 \mathrm{~A}$. Table 9 shows the means of correct RTs to left-oriented targets in trial $n$ categorized by the target-defining dimension in trial $n-1$. A two-way ANOVA was performed with main terms of dimension in trial $n-1$ and response in trial $n$, and both main effects were significant $[F(2,30)=6.43$, $p<.005$, and $F(1,15)=12.16, p<.005$, respectively]. The two-way interaction was not significant $(F<1)$. Planned comparisons showed significant differences between orientation trials and other trials [orientation and color, $F(1,30)=4.34, p<.05$; orientation and size, $F(1,30)=$ $12.74, p<.005$; orientation and average of color and size, $F(1,30)=10.65, p<.005]$. There was a clear ITF effect of $28.6 \mathrm{msec}$, on average, across target definitions and responses.

Counting task. Mean correct RTs for trials with leftoriented targets are shown in Table 10. A two-way ANOVA was performed with main terms of target definition and response (six or seven), but both of the main effects and the interaction were not reliable (all $p$ s $>.3$ ). There was no advantage of RTs in the within-dimension condition, relative to those in the cross-dimension condition.

Table 10 also shows the error rates for each condition. A two-way (target definition $\times$ response) ANOVA showed that both of the main effects and the interaction were not significant (all $p \mathrm{~s}>.19$ ). Error rates were constant across all conditions and responses, suggesting that the participants did not tend to make a speed-accuracy tradeoff.

For the cross-dimension condition, ITF was analyzed by the same method as that in Experiment 1A. Table 11 shows the means of correct RTs to left-oriented targets in trial $n$ categorized by the target-defining dimension in trial $n-1$. A two-way ANOVA was performed with the main terms of target-defining dimension in trial $n-1$ and response in trial $n$. The main effect of dimension was significant $[F(2,30)=13.76, p<.0001]$, but the main effect of response and the target definition $\times$ response interaction were not significant (both $F \mathrm{~s}<1$ ). Planned comparisons showed significant differences between orientation trials and color trials $[F(1,30)=10.10, p<$ $.005]$, orientation trials and size trials $[F(1,30)=27.08$, $p<.0001]$, and orientation trials and average of color and size $[F(1,30)=20.49, p<.0001]$. There was a clear ITF effect of $101.9 \mathrm{msec}$ on average, across target definitions and responses.

\section{Discussion}

The results concerning WDF in this experiment were straightforward: WDF was observed only in the subitizing task. This provides converging evidence in support of the attentional limitation account. When no serial guidance of attention is required, as in the subitizing task, prior knowledge of the target feature is useful, but serial guidance of attention in the counting task could not be prioritized by this knowledge.

Experiment 3 also showed that ITF is sensitive to responses but that, inconsistent with my prediction that discrimination responses would eliminate ITF, ITF was observed in both tasks, even though they both required numerosity discrimination. In Experiment $1 \mathrm{~B}$ and in the texture boundary discrimination task in Experiment 2, there was no ITF effect when the participants made discrimination responses. I therefore suggest that numerosity discrimination may be mediated by a mechanism similar to that which mediates detection. Numerosity discrimination is not a choice between two clearly categorized alternatives, like discrimination between horizontal or vertical orientations of a texture boundary. Rather, the number of elements is represented as the level of activation in a response selection process, like the presence of targets in the detection task.

\section{GENERAL DISCUSSION}

\section{Summary of Results}

In this study, WDF and ITF were observed in a variety of visual tasks-visual search, texture segregation, and visual enumeration-clearly showing that these dimension-weighting effects are not specific to visual search tasks but are a more general effect. ${ }^{4}$

Previous studies assumed that WDF and ITF are mediated by the same mechanism (Cohen \& Magen, 1999; Found \& Müller, 1996; Müller et al., 1995), but in the present experiments, the two effects were doubly dissociated, as is shown in Table 12. In the texture discrimination task, WDF occurred, but ITF did not; in the counting task, WDF did not occur, but ITF did. This provides clear evidence that the two effects are independently mediated by different processes.

Table 10

Mean Correct Reaction Times (RTs, in Milliseconds) and Error Rates (PEs, Percentages) for the Counting Task in Experiment 3

\begin{tabular}{cccccc}
\hline & \multicolumn{4}{c}{ Response } \\
\cline { 2 - 3 } \cline { 5 - 6 } Target Definition & \multicolumn{2}{c}{ Six Targets } & & \multicolumn{2}{c}{ Seven Targets } \\
\cline { 2 - 5 } \cline { 5 - 6 } & RT & PE & & RT & PE \\
\hline Within-dimension & $1,301.9$ & 3.13 & & $1,328.8$ & 3.91 \\
Cross-dimension & $1,316.0$ & 2.83 & & $1,332.1$ & 4.59 \\
\hline
\end{tabular}


Table 11

Mean Correct Reaction Times (RTs) in Trial $n$ and Intertrial Facilitation (ITF; Both in Milliseconds) for Orientation-Defined Targets as a Function of the Target-Defining Dimension in the Previous Trial $(n-1)$ in the Counting Task of Experiment 3

\begin{tabular}{lrrrrr}
\hline \multirow{2}{*}{$\begin{array}{l}\text { Target-Defining Dimension } \\
\text { of Trial } n-1\end{array}$} & \multicolumn{2}{c}{ Six Targets } & & \multicolumn{2}{c}{ Seven Targets } \\
\cline { 2 - 3 } & RT & ITF & & RT & ITF \\
\hline Orientation & $1,232.6$ & & & $1,266.2$ & \\
Color & $1,322.8$ & $90.2^{*}$ & & $1,330.7$ & $64.5 \dagger$ \\
Size & $1,372.3$ & $139.7^{*}$ & & $1,379.6$ & $113.4^{*}$ \\
Average of color and size & $1,347.5$ & $114.9^{*}$ & & $1,355.1$ & $88.9^{*}$ \\
\hline
\end{tabular}

$* p<.05 . \quad \dagger .05<p<.10$.

\section{Within-Dimension Facilitation}

When compound search tasks were used (Experiment 1A), no WDF was observed. This pattern is consistent with both the response account (Cohen \& Magen, 1999) and the attentional limitation account, but results in the subsequent experiments did not support the response account. First, WDF was still observed when texture discrimination within a single feature dimension was required (Experiment 2). Second, WDF was eliminated in the counting task (Experiment 3), despite the fact that it was observed in the subitizing task, in which the same numerosity responses were required. These results indicate that WDF is not affected by processes involved in response mechanisms.

Dimension-weighting accounts, which assume that topdown modulation of a known feature dimension facilitates processing (Müller et al., 1995), do not fully explain these results, because WDF was eliminated under both compound search and counting tasks. However, when focal attention to a spatial region was not involved, WDF was observed, not only in the simple detection task (Experiment 1A), but also in the texture detection task (Experiment 2). This is consistent with the explanation that responses are directly elicited on the basis of activation of a feature-specific saliency map, without involving focal attention. The present study demonstrated that such direct responses can occur not only in detection tasks, but also in texture discrimination and subitizing tasks. Direct responses are based not only on the presence or absence of peaks of activation on the feature-specific saliency map, but also on quantitative (e.g., number of targets) or even qualitative (e.g., orientation of texture boundaries) characteristics of activation on the map. Therefore, knowledge of the target-defining feature is useful for computation of activation on the saliency map, affecting a wider range of responses than does simple detection.

When focal attention was engaged, as in the compound search and counting tasks, there was no WDF. This is consistent with the attentional limitation account, in which spatial attention is guided by saliency signals on the master map, activation of which are unweighted by knowledge of target-defining features (Kumada, 1999; Theeuwes, 1991, 1992). Kumada suggested that knowledge of targetdefining features could modulate activation in the later stages, after saliency signals are computed and trans- mitted to the master map. This would explain why explicit knowledge of the target-defining feature dimension had no effect on control of spatial attention in the compound search and counting tasks. Such a staging of processes may enforce cognitive impenetrability between the spatial attention system and the weighted featureprocessing modules. Such impenetrability may have evolved to ensure the rapid allocation of spatial attention to salient objects in a visual scene or the detection of salient objects irrespective of prior knowledge or expectation.

\section{Intertrial Facilitation}

ITF was correlated with type of response: It disappeared when the participants made discrimination responses, but it was observed with detection and enumeration responses. Thus, ITF does not depend on whether focal attention is allocated or not. Some previous studies have shown ITF under discrimination tasks (Cohen \& Magen, 1999, Experiment 4; Found \& Müller, 1996), in which participants responded to feature values of targets that lay along the target-defining feature dimension. In the present study, ITF was not observed when discrimination response features were independent of the targetdefining feature dimension in Experiments $1 \mathrm{~B}$ and 2. In both of these experiments, ITF failed to appear only when the to-be-reported attribute was independent of the target-defining feature dimension. In other words, ITF is seen whenever response processing does not need to refer to other processes for to-be-reported attributes.

The nature of ITF is satisfactorily explained by featurebased response selection models (Cohen \& Magen, 1999; Cohen \& Shoup, 1997; Mordkoff \& Yantis, 1993). These models assume that each feature module has its own response selection processes. Intertrial priming of response selection processes in each feature module provides an advantage in the subsequent trial for targets that are defined by the same feature dimension. On the other hand, in the compound search task and in the texture discrimination task, in which responses are elicited on the basis of a to-be-reported attribute that is different from the target-defining feature dimension, there is no effect of intertrial priming, because the response selection process in each feature module is not used when responding to the to-be-reported attribute.

Table 12

Summary of Results for Within-Dimension Facilitation (WDF) and Intertrial Facilitation (ITF; Both in Milliseconds) in Experiments $1 \mathrm{~A}, 1 \mathrm{~B}, 2$, and 3

\begin{tabular}{lcc}
\hline \multicolumn{1}{c}{ Task } & WDF & ITF \\
\hline Visual search (Experiments 1A and 1B) & & \\
$\quad$ Simple search & $29.8^{*}$ & $28.2^{*}$ \\
$\quad$ Compound search & -4.7 & 14.4 \\
Texture segregation (Experiment 2) & & \\
$\quad$ Detection & $29.6^{*}$ & $25.9^{*}$ \\
$\quad$ Discrimination & $50.9^{*}$ & 7.4 \\
Enumeration (Experiment 3) & & \\
$\quad$ Subitizing & $56.6^{*}$ & $28.6^{*}$ \\
Counting & 8.7 & $101.9^{*}$ \\
\hline
\end{tabular}

${ }^{*} p<.05$. 
It is important to note that the degree of focal attention to targets was independent of ITF. Previous models of visual search have assumed that information about visual objects is registered in "object files" by focusing spatial attention on the objects (Treisman, 1988; Treisman \& Gelade, 1980), but the information that enables ITF may be registered in a different form from the "object files." I suggest that the feature modules that were just previously used for coding responses are still sufficiently activated to prime the selection of the next response. More functionally, it may be that feature information used for a previous "action" is registered and thus is available to facilitate selection of action to the same object again, independent of the degree of attention focused on it.

\section{Conclusion}

Visual tasks performed on the basis of feature dimensions are mediated by two types of dimension weighting, each occurring independently. One is knowledge-based dimension weighting, which is explicitly activated by knowledge of the target-defining feature dimension, irrespective of the type of response. The other is responsebased dimension weighting, the implicit priming of feature dimensions that were used for responding in the preceding trial. Such priming is independent of the allocation of focal attention. Distinguishing these two influences allows us to reconcile apparently contradictory accounts of WDF and ITF in visual search tasks: the dimension-weighting account (Found \& Müller, 1996; Müller et al., 1995) and the response account (Cohen \& Magen, 1999). In addition, this study has shown that knowledge-based dimension weighting is not globally available for guidance of spatial attention but is limited to certain processes and modules.

\section{REFERENCES}

Broadbent, D. E. (1958). Perception and communication. London: Pergamon.

Cohen, A., \& MAgen, H. (1999). Intra- and cross-dimensional visual search for single-feature targets. Perception \& Psychophysics, 61, 291-307.

Cohen, A., \& Shoup, R. (1997). Perceptual dimensional constraints on response selection processes. Cognitive Psychology, 32, 128-181.

Duncan, J. (1985). Visual search and visual attention. In M. I. Posner \& O. S. M. Marin (Eds.), Attention \& performance XI (pp. 85-106). Hillsdale, NJ: Erlbaum.

Found, A., \& Müller, H. J. (1996). Searching for unknown feature targets on more than one dimension: Investigating a "dimensionweighting" account. Perception \& Psychophysics, 58, 88-101.

Kahneman, D., Treisman, A., \& Burkell, J. (1983). The cost of visual filtering. Journal of Experimental Psychology: Human Perception \& Performance, 9, 510-522.

Kumada, T. (1999). Limitations in attending to a feature value for overriding stimulus-driven interference. Perception \& Psychophysics, 61, 61-79.

Mordkoff, J. T., \& YANTis, S. (1993). Dividing attention between color and shape: Evidence of coactivation. Perception \& Psychophysics, 53, 357-366.

Müller, H. J., Heller, D. \& Ziegler, J. (1995). Visual search for singleton feature targets within and across feature dimensions. Perception \& Psychophysics, 57, 1-17.

NothduRFT, H.-C. (1992). Feature analysis and the role of similarity in preattentive vision. Perception \& Psychophysics, 52, 355-375.
Theeuwes, J. (1991). Cross-dimensional perceptual selectivity. Perception \& Psychophysics, 50, 184-193.

Theeuwes, J. (1992). Perceptual selectivity for color and form. Perception \& Psychophysics, 51, 599-606.

Treisman, A. (1988). Features and objects: The fourteenth Bartlett memorial lecture. Quarterly Journal of Experimental Psychology, 40A, 201-237.

Treisman, A., \& Gelade, G. (1980). A feature-integration theory of attention. Cognitive Psychology, 12, 97-136.

Treisman, A., \& Sato, S. (1990). Conjunction search revisited. Journal of Experimental Psychology: Human Perception \& Performance, 16, 459-478.

TRICK, L. M., \& ENNS, J. T. (1997). Measuring preattentive processes: When is pop-out not enough? Visual Cognition, 4, 164-205.

Trick, L. M., \& Pylyshyn, Z W. (1993). What enumeration studies can show us about spatial attention: Evidence for limited capacity preattentive processing. Journal of Experimental Psychology: Human Perception \& Performance, 19, 331-351.

Trick, L. M., \& PY LYShYN, Z W. (1994). Why are small and large numbers enumerated differently? A limited-capacity preattentive stage in vision. Psychological Review, 101, 80-102.

VAN Selst, M., \& Jolicceur, P. (1994). A solution to the effect of sample size on outlier elimination. Quarterly Journal of Experimental Psychology, 47A, 631-650.

Wolfe, J. M. (1994). Guided Search 2.0: A revised model of visual search. Psychonomic Bulletin \& Review, 1, 202-238.

\section{NOTES}

1. As was noted by Duncan (1985), there are tasks that are hard to classify as simple search or compound search. One instance of such difficulty is when the target-defining feature and the to-be-reported attribute belong to the same feature dimension, as in Experiment 1 of Cohen and Magen (1999). In the present discussion, I assume that target detection can occur when a difference in the target-defining feature is detected between targets and nontargets and that further information is always needed to determine the to-be-reported feature of the target. In other words, the target-defining feature is not always based on the same information as the to-be-reported attribute, even when they belong to the same feature dimension. Therefore, I classified the tasks used in Cohen and Magen as one type of compound search task.

2. The significant main effect of number of elements needs to be explained. RTs were longer for 10-element displays than for 1-element displays in all three target definition conditions (by $89.2 \mathrm{msec}$ in the control condition, $98.6 \mathrm{msec}$ in the within-dimension condition, and $109.4 \mathrm{msec}$ in the cross-dimension condition). This translated to search rates in the range of 9.9-12.2 msec per additional item, times that are quite small if we attribute them to strictly serial search processes, but additional nontargets may also increase the time to search for featuredefined targets in a compound search task. This increase, referred to as filtering cost, is known to be obtained in compound search tasks, but not in simple search tasks (Kahneman, Treisman, \& Burkell, 1983). Kahneman et al. argued that the filtering cost could be explained by competition between object files for attentional resources. The effect of number of elements in this experiment should be interpreted as a filtering cost.

3 . An anonymous reviewer suggested that both the target-defining feature and the to-be-reported attribute might use the same orientation response selector for orientation target trials and that this might explain why no ITF was found. Therefore, as the reviewer suggested, I further analyzed the ITFs for color- and size-defined targets. For both kinds of targets, the ITF was not reliable: [color, $2.5 \mathrm{msec}, p>.8$; size, $-14.6 \mathrm{msec}$, $p>$.2]. This clearly suggests that the same-response-selector account is an unlikely explanation of the absence of ITF in orientation targets.

4. This study does not suggest that the visual tasks used in this study are mediated by exactly the same processes. I suggest that these tasks commonly use feature maps and response selection processes, which involve dimension-weighting effects. There may be specif ic processes for each task. However, these residual processes are not crucial for explaining dimension-weighting effects.

(Manuscript received July 7, 1999;

revision accepted for publication August 24, 2000.) 